\title{
Vortex Lattice Melting in Multilayers with Variable Anisotropies
}

\author{
P. Koorevaar, P. H. Kes, A. E. Koshelev, and J. Aarts \\ Kamerlingh Onnes Laboratory, Leiden University, P.O. Box 9506, 2300 RA Leiden. The Netherlands
}

(Received 6 August 1993)

\begin{abstract}
Vortex lattice melting is investigated by transport measurements on $\mathrm{NbGe/Ge}$ multilayers as a function of Ge thickness, which controls the anisotropy of the system. Considerable changes are found between Ge thicknesses of 2 and $4 \mathrm{~nm}$. For low anisotropies the melting line for the multilayers is indistinguishable from that for a single film with the same total thickness. Increasing the anisotropy, a crossover is observed from 2D melting in the full sample at low fields to 2D melting in single layers at high fields, with melting of $3 \mathrm{D}$ nature in the intermediate field range. Multilayers with high anisotropy only show the second crossover.
\end{abstract}

PACS numbers: $74.60 . \mathrm{Ge}, 74.80 . \mathrm{Dm}$

The influence of thermal disorder on the stability of the vortex lattice (VL) has been thoroughly studied in recent years. It is now well established that the VL can melt far below the mean-field transition at $B_{c 2}$. In perpendicular fields and for a two-dimensional (2D) VL with weak disorder, a Berezinskii-Kosterlitz-Thouless (BKT) [1,2] melting transition occurs, which is governed by the unbinding of thermally created dislocation pairs. Such melting was observed experimentally by several authors [3-6]. In a three-dimensional (3D) VL thermal fluctuations lead to an increasing value for the mean displacement of a flux line, $u(T)$, and according to the Lindemann criterion melting occurs when $u(T)$ becomes some fraction of the intervortex distance $a_{0}$. For isotropic conventional superconductors 3D melting generally occurs very close to $B_{c 2}$, but it is observable when $\kappa$ is high [7]. Anisotropy in the VL can substantially lower the $3 \mathrm{D}$ melting line $[8,9]$, which is one of the reasons why it is especially pronounced in high $-T_{c}$ materials.

Layered anisotropic materials are predicted to show complex melting behavior [10,11], since, apart from the melting line $B_{m}(T)$, there is also a decoupling line $B_{D C}(T)$. Above $B_{D C}(T)$ vortex segments is adjacent layers are effectively decoupled. The two curves intercept at a characteristic point $B_{D}\left(T_{D}\right)$. For fields above $B_{D}$, decoupling occurs at lower temperature than VL melting of the 2D individual layers, and the melting transition for the layered material should be close to the melting transition for the individual layers at $T_{2 \mathrm{D} \text {,ind }}^{m}(B)$. For fields below $B_{D}$ decoupling occurs at temperatures above the melting line, which is now of 3D nature. Furthermore, it was recently argued by Daemen et al. [12] that, because thermal fluctuations of the vortices induce phase differences across the layers, the anisotropy factor $\gamma$ is both temperature and field dependent. This influences the melting line in the $3 \mathrm{D}$ regime.

Additionally, we will show experimentally that layered materials with both a small anisotropy and a small total sample thickness $d_{\text {tot }}$ show a finite size effect. Below $B_{D}$ the melting is in principle $3 \mathrm{D}$. However, for a sample of thickness $d_{\text {tot }}$ the energetically most favorable tilt deformation has a wavelength $\pi / d_{\text {tot }}$ and at fields smaller than a typical field $B_{\mathrm{cr}}$, the energy associated with this tilt de- formation becomes larger than the melting temperature $T_{2 \mathrm{D} \text {,full, }}^{m}$ corresponding to $2 \mathrm{D} \mathrm{VL}$ melting of vortices straight over the full sample thickness. For fields below $B_{\text {cr }}$ the layered material then again shows $2 \mathrm{D} \mathrm{VL}$ melting, but now at a temperature corresponding to $d_{\text {tot }}$ (provided $d_{\text {tot }}$ is small enough for $2 \mathrm{D}$ melting to occur). Since the BKT melting temperature scales with the effective length of the vortices, the $2 \mathrm{D}$ melting lines for fields above $B_{D}$ and below $B_{\text {cr }}$ are clearly different.

The $\mathrm{NbGe/Ge}$ multilayer system is well suited to test these new ideas. As was shown in [5], thin NbGe layers show 2D VL melting in agreement with the BKT theory. By changing the Ge layer thickness in the multilayers between 2 and $6 \mathrm{~nm}$, the anisotropy varies in such a way that crossovers in the melting transitions can be demonstrated, as well as the effects of finite sample thickness. We also found indications for the field dependence of $\gamma$ as predicted in Ref. [12].

Samples were prepared by dc magnetron sputtering at an Ar pressure of $5 \times 10^{-3}$ mbar on Si substrates at room temperature, in an UHV system with a base pressure of $10^{-9}$ mbar. Sputtering rates were calibrated by ion scattering [Rutherford backscattering (RBS)] on single films of $\mathrm{Nb}$ and $\mathrm{Ge}$ and by stylus measurements on single NbGe layers. From microprobe analysis and RBS, the exact $\mathrm{NbGe}$ composition was found to be $\mathrm{Nb}_{63} \mathrm{Ge}_{37}$. Xray diffraction showed both the $\mathrm{NbGe}$ and $\mathrm{Ge}$ to be amorphous. The layered structure of the multilayers was confirmed by RBS.

Below we discuss current-voltage $(I V)$ characteristics and ac resistivity $\left(\rho_{\mathrm{ac}}\right)$ for two $\mathrm{NbGe}$ single layers of thickness 18 and $90 \mathrm{~nm}$ (called S18 and S90), and for four $\mathrm{NbGe} / \mathrm{Ge}$ multilayers, consisting of $5 \mathrm{NbGe}$ layers of thickness $d_{s}=18 \mathrm{~nm}$ separated by Ge layers of thickness $d_{i}$. Multilayers were prepared with $d_{i}=2.2,2.6,3.0$, and $6.0 \mathrm{~nm}$ (called $\mathrm{M} 22$, etc.). All samples had $60 \mathrm{~nm}$ protective Ge top and bottom layers. $T_{c}$ was determined from the midpoints of the resistance transitions; see Table I. $\Delta T_{c}$ was typically $30 \mathrm{mK}$. For multilayer M22 we could estimate the anisotropy factor $\gamma_{0}\left(=\xi_{a b} / \xi_{c}\right.$, with $\xi_{a b}, \xi_{c}$ the coherence length parallel and perpendicular to the layers), since it showed the well known crossover in $B_{c 2 \|}$ from 3D behavior close to $T_{c}$ to 2D behavior at 
TABLE I. The derived sample parameters.

\begin{tabular}{cccccc}
\hline \hline Sample & $\begin{array}{c}T_{c} \\
(\mathrm{~K})\end{array}$ & $\begin{array}{c}B_{c 2}(0) \\
(\mathrm{T})\end{array}$ & $\begin{array}{c}\xi_{a b}(0) \\
(\mathrm{nm})\end{array}$ & $\kappa$ & $\gamma_{0}$ \\
\hline S90 & 3.16 & 5.63 & 6.36 & 78.5 & $\ldots$ \\
S18 & 2.93 & 4.82 & 6.87 & 73.3 & $\ldots$ \\
M22 & 2.94 & 4.91 & 6.80 & 83.4 & 4.6 \\
M26 & 2.95 & 6.35 & 5.98 & 97.4 & 5.8 \\
M30 & 2.91 & 6.03 & 6.14 & 96.3 & 7.3 \\
M60 & 2.94 & 7.21 & 5.62 & 104.7 & 42 \\
\hline \hline
\end{tabular}

lower temperatures. Both the slope $S_{\|}=-\partial B_{c 2 \|} / \partial T$ at $T_{c}$ and the crossover temperature $T^{\mathrm{cr}}$ can be used to find $\xi_{c}$, yielding $\gamma_{0}=4.6$. All other multilayers showed $2 \mathrm{D}$ behavior in $B_{c 2}$ for all temperatures. For these, we estimated $\gamma_{0}$ by using the relation [13] $\gamma_{0}^{2}=(\alpha / \Lambda)^{2}$ $\times \exp \left(d_{i} / d_{0}\right)$, with $\alpha$ a constant and $\Lambda$ the multilayer periodicity. Inserting a tunneling length of $d_{0} \approx 0.8 \mathrm{~nm}$ for amorphous Ge [13] and $\gamma_{0}$ from M22 yields $\gamma_{0}$ for the other multilayers, shown in Table I. These estimates only take into account the Josephson coupling, neglecting magnetic coupling.

As shown in Refs. [5, 14], the resistive transition of thin $\mathrm{NbGe}$ layers in perpendicular field substantially broadens due to VL melting. This was analyzed by comparing the ac resistivity $\rho_{\text {ac }}$ to the flux-flow resistivity $\rho_{\mathrm{FF}}$, which was determined from $I V$ characteristics and defined as $\partial V / \partial I$ in a current regime where the vortices move uniformly with velocity $v=E / B$. The melting field $B_{m}$ is found as the field at which $\rho_{\text {ac }}$ merges with $\rho_{\text {FF. }}$. The reasons for this choice for $B_{m}$, instead of, e.g., $\rho_{\mathrm{ac}} \rightarrow 0$, were discussed extensively in [14]. Below $B_{m}, \rho_{\text {ac }}$ drops exponentially, while $\rho_{\mathrm{FF}}$ remains finite. We found this same characteristic behavior of $\rho_{\mathrm{ac}}(B)$ and $\rho_{\mathrm{FF}}(B)$ for both the thin monolayers and the multilayers, and used it to determine $B_{m}$.

The experimental parameters for $\rho_{\text {ac }}$ were ac driving currents of typically $0.05 \mathrm{~A} / \mathrm{cm}^{2}$, at a frequency of 120 $\mathrm{Hz}$, while $\rho_{\mathrm{FF}}$ was determined at a voltage corresponding to a flux line velocity $v \approx 0.1 \mathrm{~m} / \mathrm{s}$. Typical results for $\rho_{\text {ac }}$ and $\rho_{\text {FF }}$ at $T=2.1 \mathrm{~K}$ for several multilayers are shown on a linear scale in Fig. 1(a), and on a semilogarithmic scale in Fig. 1(b), where we also show the result for S18. We observe [Fig. I (a)] that just below $B_{c 2}$ the $\rho_{\mathrm{FF}}(B)$ is linear over a relatively large $B$ interval, as expected when fluctuations are neglected [15]. Extrapolating this behavior to $\rho_{n}$ defines $B_{c 2}$, as illustrated in Fig. $1(\mathrm{a})$. All samples show a pronounced rounding of $\rho$ close to $B_{c 2}$, which becomes stronger for higher $T$. For monolayers it was shown that this is due to fluctuations [14], and this will also be of importance in the multilayers. The definition of the melting field $B_{m}$ is made apparent in the logarithmic plot of Fig. 1(b). The figure also makes clear that $B_{m} / B_{c 2}$ is lowest for $\mathrm{S} 18$ and increases for the multilayers with decreasing $d_{i}$. The implications are discussed below, where we systematically give the $B-T$ phase diagrams for all samples.
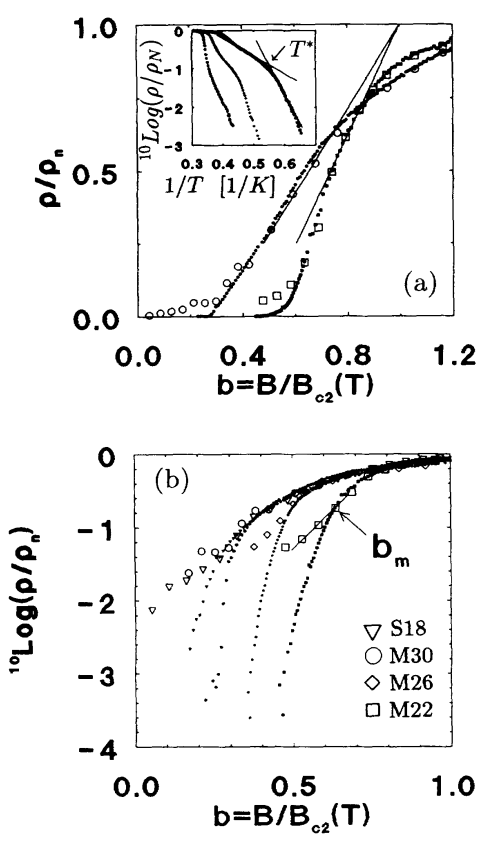

FIG. 1. (a) $\rho_{\mathrm{FF}}$ (open symbols) and $\rho_{\text {ac }}$ (filled symbols) vs $b$ at $T=2.10 \mathrm{~K}$ for M30 (O) and M22 ( $\square$ ) on a linear scale. In (b) the same data and the results for M26 $(\diamond)$ and $\operatorname{S18}(\nabla)$ are shown on a semilogarithmic plot. The construction for $B_{c 2}$ is shown in (a) and for $B_{m}$ in (b). The inset shows $\rho_{\text {ac }}$ vs $T$ data in Arrhenius fashion for $\mathrm{S} 18$ at fields $B$ of (from left to right) $0.1,0.4$, and $0.95 \mathrm{~T}$. The points $T^{*}(B)$, defined by the construction shown for $B=0.95 \mathrm{~T}$, coincide with the $B_{m}(T)$ line constructed via the $\rho_{\text {ac }}=\rho_{\text {FF }}$ method; see Fig. 2 (a).

Starting with the single layer results [Fig. 2(a)], we can fit $B_{c 2}(T)$ to the theoretical expression for $s$-wave superconductors [16], which is shown by the upper line. Good agreement is found, yielding $\boldsymbol{B}_{\boldsymbol{c} 2}(0)$ (see Table I). Since the $\mathrm{NbGe}$ layers are weak-coupling amorphous superconductors, the experimental values for the slope $S=-\partial B_{c 2} / \partial T$ at $T_{c}$ and for $\rho_{n}(0)$ can be used [17] to determine $\kappa\left(=3.54 \times 10^{4}\left[\rho_{n}(0) S\right]^{1 / 2}\right)$ and $\lambda_{a b}(0)$ $\left[=1.63 \kappa \xi_{a b}(0)\right]$. All parameters thus determined are in accordance with previously reported values for $a$ $\mathrm{Nb}_{1-x} \mathrm{Ge}_{x}$.

Next we concentrate on the melting fields for the monolayers as shown in Fig. 2(a). The BKT melting criterion for a $2 \mathrm{D} V \mathrm{~L}$ reads $[1,2]$

$$
A c_{66} a_{0}^{2} d / k_{B} T^{\mathrm{BKT}}=4 \pi .
$$

The shear modulus $c_{66}$ is given by $c_{66}=\left[B_{c}(t)^{2} / 4 \mu_{0}\right]$ $\times b\left(1-0.58 b+0.29 b^{2}\right)(1-b)^{2}[18], b=B / B_{c 2}, t=T /$ $T_{c}$, and $A \approx 0.64$ is a renormalization factor for $c_{66}$ due to nonlinear lattice vibrations $[2,19]$. The BKT melting line $T^{\mathrm{BKT}}(B)$ crucially depends on the thickness $d$ of the 2D sample. Figure 2(a) shows that the experimental data for the melting curves for both $\mathrm{S} 18$ and $\mathrm{S} 90$ agree nicely with the theoretical expression for the BKT melting, Eq. (1), i.e., for $d=18 \mathrm{~nm}$ and $d=90 \mathrm{~nm}$, respectively. 

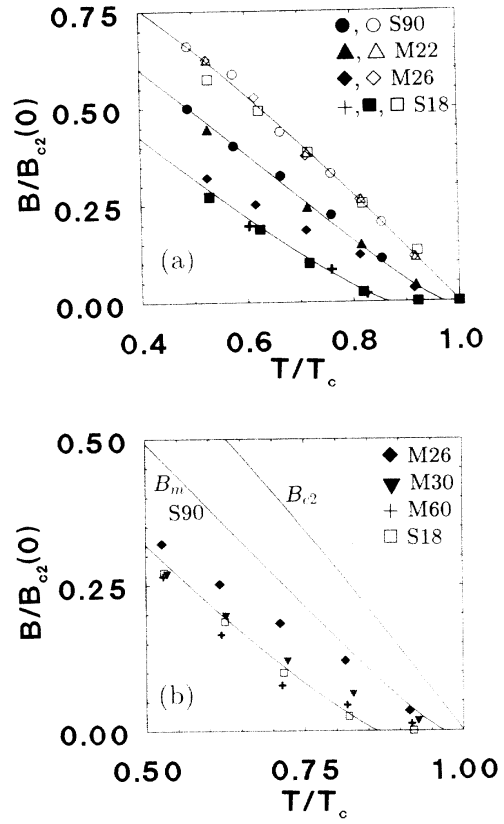

FIG. 2. Phase diagram for monolayers and multilayers. (a) $B_{c 2}(T) / B_{c 2}(0)$ (open symbols) for S18 (ם), S90 (O), M22 ( $\left.\triangle\right)$, and M26 $(\diamond)$, compared with the theoretical expectation (upper solid curve) from Ref. [16]. The $B_{m}(T) / B_{c 2}(0)$ data (filled symbols) are also shown, together with the theoretical expectation [Eq. (1)] for $B_{m}(T)$ for $\mathrm{S} 18$ (lower curve) and $\mathrm{S} 90$ (middle curve). The $(+)$ symbols indicate $T^{*}(B) / T_{c}$ data for S18 constructed from the Arrhenius plot shown in the inset of Fig. I (a). In (b) the $B_{m}$ data for M30 ( $\left.\nabla\right)$ and M60 (+) are shown, together with replotted data for M26 $(\bullet), S 18(\square)$, and the theoretical curves described under (a).

A comment on the role of pinning induced disorder in the VL on the BKT melting is needed. Yazdani et al. [6] showed experimentally that in $a-\mathrm{MoGe}$ films the BKT melting is not strongly influenced by pinning when the order in the VL, measured by the transverse correlation length $R_{c}$ [20], is sufficiently large $\left(R_{c} / a_{0} \geq 10\right)$. Strong deviations from BKT behavior are observed in small fields and in very thin films $(6.0 \mathrm{~nm})$, when $R_{c} / a_{0}$ becomes of order unity $[6,21]$. In our $\mathrm{NbGe}$ samples, where critical current densities are typically a factor of 100 less than in MoGe, the role of pinning is even smaller. Analyzing critical current measurements with $2 \mathrm{D}$ collective pinning theory (see, e.g., [17]) we determined that for S18 at $T=1.55 \mathrm{~K}$, where the role of disorder should be most predominant, $R_{c} / a_{0}$ was about 18 for $B$ just below $B_{m}$. This result is in accordance with previous estimates for thicker films [15], for which $R_{c} / a_{0}\left(\propto d^{1 / 2}\right)$ is even larger (here $d$ denotes the film thickness). Therefore we believe that the BKT melting fields in all our samples are not markedly influenced by the pinning. The nice agreement between theory and experiment shown in Fig. 2(a) confirms this.

Next we turn to the multilayers. The experimental $B_{c 2}(T)$ data again fit standard theory [16], as shown for
M22 and M26 in Fig. 2(a). Concentrating on $\tilde{b}_{m}$ $=B_{m} / B_{c 2}(0)$ for M22 we see that it practically coincides with the results for the almost equally thick sample S90. indicating that in M22 the vortices in the different layers are strongly coupled and are straight over the whole sample on the melting line, which in our notation is the $T_{2 \mathrm{D} \text {,full }}^{m}(B)$ line. Close to $T_{c}, \tilde{b}_{m}(t)$ for M26 also coincides with the results for $\mathrm{S} 90$ [i.e., the $T_{2 \mathrm{D} \text {, full }}^{m}(B)$ line]. However, at lower $t$ deviations arise, and $\tilde{b}_{m}(t)$ shifts closer to the $\tilde{b}_{m}(t)$ curve for the $18 \mathrm{~nm}$ monolayer. This indicates that on the $T_{2 \mathrm{D} \text {, full }}^{m}(B)$ line at high $t$ and low $\bar{b}_{m}$ the interlayer vortex coupling in M26 is relatively strong, yielding straight vortices over the whole sample, and melting of 2D character. At lower $t$ on the $T_{2 \mathrm{D} \text {,full }}^{m}(B)$ line, tilt deformations in the VL can exist, and the melting has a 3D character. So, S90 shows 2D melting whereas M26 shows a 3D type of melting, even though the total sample thicknesses hardly differ. The reason is that the layered structure of M26 strongly reduces the tilt modulus $c_{44}$, favoring tilt deformations.

The crossover in the melting behavior sets in when the typical energy of the most favorable tilt deformation $E_{\mathrm{TD}}$, with wave vector $\pi / d_{\text {tot }}$, becomes comparable to $T_{2 \mathrm{D} \text {, full. }}^{m}$ Estimating $E_{\mathrm{TD}}=c_{44}\left(\pi / d_{\mathrm{tot}}\right)^{2}\left(a_{0}^{2} d_{\mathrm{tot}}\right) u_{z}^{2}$, with $u_{z}$ the characteristic displacement of a vortex due to tilt deformations, one needs to take into account the dispersion of $c_{44}\left(k_{\perp}, k_{z}\right)$. The most relevant wave vector $k_{\perp}$ is expected to be near the Brillouin zone radius $K_{0}$, and in circular approximation $K_{0}=\left(4 \pi B / \phi_{0}\right)^{1 / 2}$ [11]. Furthermore, we estimate [8] $c_{44}\left(K_{0}, \pi / d_{\text {tot }}\right) \approx\left(B^{2} / \mu_{0}\right)\left(1 / \gamma^{2}\right)(1-b) /$ $\lambda^{2} K_{0}^{2}$. According to the Lindemann criterion, the mean displacement of a vortex at the melting line equals $c_{1} a_{0}$, with $c_{l} \approx 0.1$. Using this as upper bound for $u_{z}$ and equating $E_{\text {TD }}$ to $T_{2 \mathrm{D} \text {, full }}^{m}$ leads to a characteristic crossover field

$$
B_{\mathrm{cr}}\left(1-B_{\mathrm{cr}} / B_{c 2}\right)=\frac{1}{\gamma^{2}} \frac{1}{d_{\mathrm{tot}}^{2}} \frac{16 \pi^{3} c_{l}^{2} \phi_{0}}{A}
$$

We estimate $B_{\mathrm{cr}}$ from Fig. 2(a) by intercepting the extrapolated $3 \mathrm{D}$ melting line with the $T_{2 \mathrm{D}, \text { full }}^{m}(B)$ line. We find $B_{\mathrm{cr}}=0.64 \mathrm{~T}\left(\tilde{b}_{\mathrm{cr}}=0.1\right.$ at $\left.t=0.86\right)$, which yields $\gamma=10.6$, in qualitative agreement with the estimation $\gamma_{0}=5.8$ discussed above.

We discuss $\tilde{b}_{m}(t)$ for M30 and M60, shown in Fig. 2(b). Even for the highest $t$ measured, $\tilde{b}_{m}(t)$ for these multilayers never coincides with the result for S90. This shows that $B_{\mathrm{cr}}$ for these multilayers is very low, in agreement with the strong $\gamma$ dependence of $B_{\mathrm{cr}}$ according to Eq. (2). So at high $t$ we only observe a $3 \mathrm{D}$ type of melting. Furthermore, at higher $t$ there is a clear difference in $\tilde{b}(t)$ for S18, M 30, and M60, which tends to disappear at low $t$, where all $\tilde{b}(t)$ curves converge to the result for $\mathrm{S} 18$ [22]. Melting at low $t$ is therefore of individual layers, and of a 2D nature.

According to theory $[10-12]$ the $2 \mathrm{D}$ to $3 \mathrm{D}$ transition in the melting line should occur at the point $B_{D}\left(T_{D}\right)$, where the coupling energy of vortices in adjacent layers, $E_{J}$, is 
of the order of the melting temperature for the individual layers, $T_{2 D \text {,ind }}^{m}$, or, equivalently, where the line $B_{D C}(T)$ intercepts the $T_{2 \mathrm{D} \text {,ind }}^{m}(B)$ line. A self-consistent analysis, taking into account the $T$ and $B$ dependence of $\gamma$ due to fluctuation induced phase differences across the layers, shows that for multilayers with moderate anisotropy factor $\left[\xi_{a b}(0) / d \ll \gamma_{0} \ll \lambda_{a b}(0) / d\right]$ the decoupling line $B_{D C}(T)$ is given by [12]

$$
B_{D C}(T) \approx \frac{\phi_{0}^{3}}{4 \pi^{2} \mu_{0} k_{B} T d e \lambda_{a b}^{2} \gamma_{0}^{2}} \quad(e=2.718 \ldots),
$$

which can be used at the measured point $B_{D}\left(T_{D}\right)$. Experiment indicates for M30 that $\tilde{b}_{D} \approx 0.12$ at $t=0.72$, which yields a predicted $\gamma_{0}=7.1$, in remarkable agreement with the estimation $\gamma_{0}=7.3$ discussed above. For M60 $\gamma_{0}(\approx 42)$ is comparable to $\lambda_{a b}(0) / d(\approx 50)$, and Eq. (3) is not valid. When using $\tilde{b}_{D}=0.057$ at $t=0.75$, Eq. (3) yields $\gamma_{0} \approx 10$, much smaller than expected.

As a matter of fact, the difference in $\tilde{b}_{m}(t)$ for M30 and M60 is relatively small, taking into account the expected large differences in $\gamma_{0}$ assuming Josephson coupling. This might indicate that for M60 magnetic coupling of the vortices is important as well, leading to a lower effective value for $\gamma_{0}$. On the other hand, the $B_{D}\left(T_{D}\right)$ point for $\mathrm{M} 60$ is also poorly described by assuming magnetic coupling only [i.e., Eq. (21) of Ref. [12]], as could be expected, since the criterion $\gamma_{0} \gg \lambda_{a b} / d$ is not met.

Finally we compare our results to the ac resistivity data of White, Kapitulnik, and Beasley on $\mathrm{MoGe} / \mathrm{Ge}$ multilayers [23], who found a kink in $\rho_{\mathrm{ac}}(T)$ at $T=T^{*}(B)$ (their notation), which was interpreted as a coupling-decoupling transition of the vortices is adjacent layers. Our $\rho_{\mathrm{ac}}(T)$ for both M60 and S18 shows a similar kink at $T=T^{*}(B)$ [see Fig. 1(a)]. As shown for S18 in Fig. 2(a), all $T^{*}(B)$ data coincide with the melting lines. We therefore believe that in our case the kink signals a melting transition rather than a decoupling transition. However, for the multilayers we cannot rule out the possibility that in a certain part of the phase diagram the decoupling and melting line coincide, especially since perpendicular transport measurements on $a-\mathrm{MoGe} / \mathrm{Ge}$ multilayers [24] indicate that interlayer decoupling also can coincide with $T^{*}$. We should emphasize that the melting phenomenon can only be observed when the pin energy is large enough to prevent thermal depinning below the melting line. This implies weak disorder, i.e., large Larkin domains, which does not apply to multilayers with very thin superconducting components, as in Ref. [23].

In conclusion, we report dimensional crossovers in the VL melting for $\mathrm{NbGe} / \mathrm{Ge}$ multilayers. At high temperatures the coupling between the layers is relatively strong, leading to a 3D-like melting curve with a field dependent anisotropy, or, for low anisotropic multilayers, to straight vortices over the whole sample, yielding a 2D coupled melting curve. A crossover between these behaviors is observed. For multilayers with large $\gamma_{0}$ the melting curve approaches the melting curve for individual layers at low temperature. The field $B_{D}$ where the transition from $3 \mathrm{D}$ to $2 \mathrm{D}$ single-layer melting occurs is in qualitative agreement with recent theoretical models.

We thank Professor J. A. Mydosh for his interest and C. Zwart and M. Theunissen for experimental assistance. This work was supported by the Dutch Foundation for Fundamental Research on Matter (FOM).

[1] B. A. Huberman and S. Doniach, Phys. Rev. Lett. 43, 950 (1979)

[2] D. S. Fisher, Phys. Rev. B 22, 1190 (1980).

[3] A. F. Hebard and A. T. Fiory, Physica (Amsterdam) $109 \& 110 B, 1637$ (1982).

[4] L. Gammel, A. F. Hebard, and D. J. Bishop, Phys. Rev. Lett. 60, 144 (1988).

[5] P. Berghuis, A. L. F. van der Slot, and P. H. Kes, Phys. Rev. Lett. 65, 2583 (1990).

[6] A. Yazdani, W. R. White, M. R. Hahn, M. Gabay, M. R. Beasley, and A. Kapitulnik, Phys. Rev. Lett. 70, 505 (1993)

[7] M. Suenaga, A. K. Ghosh, Youwen Xu, and D. O. Welch, Phys. Rev. Lett. 66, 1777 (1991).

[8] A. Houghton, R. A. Pelcovits, and A. Sudbø, Phys. Rev. B 40, 6763 (1989).

[9] E. E. Brandt, Phys. Rev. Lett. 63, 1106 (1989).

[10] M. V. Feigel'man, V. B. Geshkenbein, and A. I. Larkin, Physica (Amsterdam) 167C, 177 (1990); V. M. Vinokur, P. H. Kez, and A. E. Koshelev, Physica (Amsterdam) 168C, 29 (1990).

[11] L. I. Glazman and A. E. Koshelev, Phys. Rev. B 43, 2835 (1991)

[12] L. L. Daemen, L. N. Bulaevskii, M. P. Maley, and J. Y. Coulter, Phys. Rev. Lett. 70, 1167 (1993).

[13] S. T. Ruggiero, T. W. Barbee, and M. R. Beasley, Phys. Rev. B 26, 4896 (1982).

[14] P. Berghuis and P. H. Kes, Phys. Rev. B 47, 262 (1993).

[15] A. I. Larkin and Yu. Ovchinnikov, in Non-Equilibrium Superconductivity, edited by P. N. Langeberg and A. I. Larkin (North-Holland, Amsterdam, 1986).

[16] N. R. Werthamer, E. Helfland, and P. C. Hohenberg, Phys. Rev. 147, 295 (1966).

[17] P. H. Kes and C. C. Tsuei, Phys. Rev. B 28, 5126 (1983).

[18] E. H. Brandt, Phys. Rev. B 34, 6514 (1986).

[19] S. W. de Leeuw and J. W. Perram, Physica (Amsterdam) 113A, 546 (1982).

[20] A. I. Larkin and Yu. Ovchinnikov, J. Low Temp. Phys. 34, 409 (1979)

[21] A. Kapitulnik, A. Yazdani, W. R. White, and M. R. Beasley, in Proceedings of the 1993 International ISTEC Workshop on Superconductivity, Hakodate, Kokusai, Japan (to be published); (to be published).

[22] The fact that for some temperatures $\tilde{b}_{m}$ for M60 is lower than for S18 might be caused by interlayer coupling of dislocations, favoring BKT melting. We thank D. Feinberg for pointing this out.

[23] W. R. White, A. Kapitulnik, and M. R. Beasley, Phys. Rev. Lett. 66, 2826 (1991).

[24] D. G. Steel, W. R. White, and J. M. Graybeal, Phys. Rev. Lett. 71, 161 (1993). 